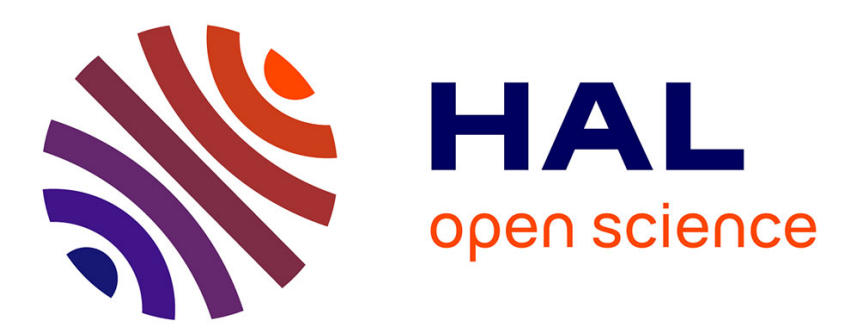

\title{
La scolarisation en milieu spécialisé des élèves avec handicap dans la littérature jeunesse
}

\author{
Lise Lemoine, Benoît Schneider
}

\section{To cite this version:}

Lise Lemoine, Benoît Schneider. La scolarisation en milieu spécialisé des élèves avec handicap dans la littérature jeunesse. Enfances \& Psy , 2019, 2 (82), pp.76-87. 10.3917/ep.082.0076 . hal-02367602

\section{HAL Id: hal-02367602 \\ https://hal.science/hal-02367602}

Submitted on 18 Nov 2019

HAL is a multi-disciplinary open access archive for the deposit and dissemination of scientific research documents, whether they are published or not. The documents may come from teaching and research institutions in France or abroad, or from public or private research centers.
L'archive ouverte pluridisciplinaire HAL, est destinée au dépôt et à la diffusion de documents scientifiques de niveau recherche, publiés ou non, émanant des établissements d'enseignement et de recherche français ou étrangers, des laboratoires publics ou privés. 


\title{
La scolarisation en milieu spécialisé des élèves avec handicap dans la littérature jeunesse
}

\section{Lise Lemoine Benoît Schneider}

\begin{abstract}
Résumé
La littérature de jeunesse consacrée aux situations de handicap peut représenter un médiateur et un support des approches pédagogiques inclusives. Pour évaluer leur potentiel à cette fin, nous avons examiné 17 ouvrages portant sur la scolarisation en milieu spécialisé issus d'un corpus de 90 livres francophones publiés au XXlè siècle dont la thématique est consacrée aux déficiences sensorielles (cécité et surdité) et motrice et aux troubles neurodéveloppementaux (trisomie et autisme). Nous proposons de (1) dégager les caractéristiques essentielles (ressenti des héros en situation de handicap, représentations du milieu spécialisé et relations institutionsfamilles), communes et par type de handicap ; (2) effectuer le parallèle fiction/réalité (inclusion et évolution de celle-ci) ; (3) pointer l'intérêt et les limites d'une reprise potentielle de ces ouvrages pour le lecteur en vue d'une sensibilisation.
\end{abstract}

\section{Mots-clés}

Handicap, littérature de jeunesse, scolarisation.

\section{Summary}

The schooling in a specialized environment of students with disabilities in children's literature

Children's literature on disability situations can be a mediator and a support for inclusive pedagogical approaches. To assess their potential for this purpose, we examined 17 books on schooling in specialized settings from a corpus of 90 French-language books published in the 21st century on sensory (blindness and deafness) and motor disabilities and neurodevelopmental disorders (trisomy and autism). We propose to (1) identify the essential characteristics (feelings of heroes in a situation of disability, representations of the specialized environment and relations between institutions and families), common and by type of disability; (2) carry out the parallel fiction/reality (inclusion and its evolution); (3) point out the interest and limits of a potential recovery of these works for the reader in order to raise awareness.

\section{Keywords}

Disability, children's literature, school.

\footnotetext{
Lise Lemoine, Maître de conférences en psychologie du développement, Université de Rennes Laboratoire LP3C - EA 1285.

Benoît Schneider, Professeur en psychologie de l'éducation, Université de Lorraine - Laboratoire 2LPN EA 7489.
} 


\section{Introduction}

Dans le contexte d'une évolution éditoriale riche et diversifiée, les livres pour enfants proposent volontiers aux jeunes lecteurs l'abord de situations difficiles ou problématiques (Schneider, Mietkiewicz, 2013 ; Schneider, 2017). Inscrits dans des récits plus ou moins réalistes, les enfants, leurs parents, les pédagogues, les thérapeutes voient abordées dans ces ouvrages des situations problèmes et des modalités de réponses en accord avec le contexte social et les normes culturelles du moment. L'apparition auX $X I X^{e}$ et $X X^{e}$ siècles d'ouvrages sur le thème du handicap a fait émerger de nouveaux usages pédagogiques des livres pour enfants (Joselin, 2013 ; Miet, Recher, 1989).

Comme le mentionne Joselin (2008, p. 6), " l'étude des représentations renseigne sur un consensus social concernant les personnages en situation de handicap et les représentations dans la littérature enfantine offrent des indices concernant la position d'une société donnée visà-vis des enfants en situation de handicap et de leurs proches $॥$.

Plutôt bien documentés malgré parfois quelques approximations, ces ouvrages parviennent, tout en pointant sans ambiguïté les déficiences, à mettre en évidence les aptitudes des enfants avec handicap et leurs capacités adaptatives (Lemoine, Mietkiewicz, Schneider, 2016). Nous proposons donc de considérer la littérature jeunesse comme un indicateur de la manière dont la question du droit à l'éducation des enfants avec handicap est approchée dans ce support culturel à destination des enfants.

La loi de 2005 a promulgué l'obligation de scolarisation de tout enfant en situation de handicap. Dans ce domaine, des progrès indéniables ont été réalisés et restent encore à faire (cf. pour exemple le récent rapport de C. Devandas-Aguilar, 2019). Ils impliquent en particulier de prendre en compte la place des familles dans leur rapport au handicap et aux institutions (thérapeutiques, médico-sociales et scolaires). Faivre (2006) a rendu compte de l'évolution de la place des parents, selon un mouvement corollaire à celui de la loi de 2005. Au milieu du $X X^{e}$ siècle, les parents, désarçonnés par le choc du handicap, doivent faire face au cloisonnement des professions médicales et sociales qui les considèrent comme pathogènes. Au fil des décennies, tous les partenaires découvrent pas à pas que les enfants, même en situation complexe de dépendance, sont éducables. Les associations de familles se sont mobilisées pour sortir leurs enfants des hôpitaux psychiatriques pour le droit à l'éducation et ont créé des institutions médicosociales, puis se sont mobilisées par la suite pour une désinstitutionalisation au profit d'une scolarisation pensée par un projet individualisé élaboré en fonction des BEP (Besoins Educatifs Particuliers) de l'enfant. Mais cette scolarisation ne va pas de soi, l'inclusion se travaille, se réfléchit avec les élèves au-delà des aménagements matériels. L'inclusion ne peut se faire que si l'enfant est accepté et trouve sa place parmi les autres élèves dans un contexte bienveillant.

Les enfants porteurs de handicap peuvent côtoyer dans les institutions scolaires qu'ils fréquentent des élèves avec un handicap sensoriel, moteur ou un trouble neurodéveloppemental, autrement dit des enfants différents dont ils ignorent le plus souvent les difficultés, les besoins spécifiques et les ressentis. De nombreux ouvrages se sont attachés à prendre en compte la diversité des types de handicaps. Ces récits fictionnels affichent clairement, le plus souvent, l'intention de contribuer à changer le regard des écoliers sur leurs pairs en situation de handicap. Ils contribuent en ce sens à une démarche plus globale à visée "inclusive ». Nous nous sommes attachés dans une publication précédente (Mietkiewiz, Lemoine, Schneider, 2017) à examiner les liens entre enfant porteur de handicap, famille et école, mais en nous centrant sur les contextes de scolarisation en milieu ordinaire, les plus explicitement centrés sur la question de l'inclusion. 
Nous proposons ici d'envisager ici les modalités selon lesquelles la question de la scolarisation en milieu spécialisé est abordée. Elle concerne encore de très nombreux enfants. Lieu de vie essentiel pour ces enfants avec handicap, le milieu de vie spécialisé est moins connu par les pairs et par la fratrie, voire par les parents eux-mêmes, du fait d'une méconnaissance par nonexpérience ; ils n'en ont souvent qu'une représentation pouvant être assez éloignée de la réalité. Les politiques inclusives tentent, de leur côté, de traiter la transition d'un type de scolarisation à l'autre. Les associations gestionnaires d'institutions créent de nouvelles modalités de scolarisation, comme des UEM (Unité d'Enseignement en Ecole Maternelle), et lorsque cela correspond au projet des enfants, une scolarisation mixte milieu ordinaire/milieu spécialisé peut se mettre en place. Mais la question de la transition et du passage implique donc bien la connaissance de l'institution spécialisée elle-même. Les ouvrages de jeunesse abordent-ils ce contexte de vie ? Selon quelles modalités ? Quel type de regard sur les enfants avec handicap et leur scolarisation nous proposent-ils?

Après avoir présenté notre méthodologie de sélection des ouvrages, nous aborderons, par type de handicap, le cadre général du contexte de scolarisation des enfants avec handicap, le type de positionnement familial et parental au regard de la scolarisation et la nature des liens institutions-familles.

\section{Méthodologie}

Le corpus du présent article fait partie d'un corpus plus large d'ouvrages publiés ou réédités au $X X X^{\mathrm{e}}$ siècle en langue française (154 livres) établi en référence à une catégorisation de handicaps avérés, voire "prototypiques » : cécité, surdité, handicap locomoteur, trisomie et autisme.

Ce corpus a été constitué à partir de la consultation des sites web dédiés à la littérature jeunesse, ceux des associations consacrées à l'enfance en situation de handicap, le réseau $\mathrm{CANOPE}^{1}$ (réseau de création et d'accompagnement pédagogiques) et les informations et publications recueillies auprès des bibliothécaires et libraires spécialisés ${ }^{2}$.

Nous avons retenu les récits fictionnels, albums et romans destinés aux enfants de 3 à 11 ans inclus (selon la mention fournie par l'éditeur, à défaut selon l'information retenue par les sites de référence en littérature jeunesse: Ricochet ${ }^{3}$, Babelio ${ }^{4}$ ) et exclu les guides de sensibilisation sans scénario narratif ou les livres à destination des enfants avec handicap euxmêmes.

Parmi ces 154 ouvrages, 90 abordent le thème de la scolarisation; 68 d'entre eux abordent une scolarisation de l'enfant-héros en milieu ordinaire ${ }^{5} ; 17$ s'attachent à des situations ou des scénarios spécifiques (manque d'information, absence de scolarité, enseignement par correspondance...) ; enfin 17 seulement abordent la scolarisation en milieu spécialisé - avec parfois une scolarisation mixte -, contexte qui fait l'objet de la présente analyse. Le tableau 1 présente la déclinaison sélective que nous avons opérée.

Parmi ces 154 ouvrages, 90 abordent le thème de la scolarisation, et 17 la scolarisation en milieu spécialisé (MS) ${ }^{6} ; 13$ parmi ces derniers abordent les liens école-famille ${ }^{7}$.

\footnotetext{
1 https://www.reseau-canope.fr/

2 «Les livres du mois » (Livres Hebdo, Bibliographie de la France).

3 http://www.ricochet-jeunes.org/

${ }^{4}$ https://www.babelio.com/

${ }^{5}$ Analyse dans Lemoine, Mietkiewicz, Schneider (2017).

${ }^{6}$ Dans la suite du texte, nous utiliserons les sigles suivants : MS : Milieu spécialisé ; MO : Milieu Ordinaire

${ }^{7}$ L'analyse des ouvrages concernant la scolarisation des enfants en $\mathrm{MO}$ a donné lieu à une publication spécifique (Lemoine, Mietkiewicz, Schneider, 2017).
} 
Tableau 1: Nombre d'ouvrages en fonction des thématiques traitées et du type de handicap.

\begin{tabular}{|l|c|c|c|c|c|c|}
\hline $\begin{array}{l}\text { Type de handicap } \\
\text { Thématiques }\end{array}$ & Cécité & Surdité & Locomoteur & Trisomie 21 & Autisme & Total \\
\hline $\begin{array}{l}\text { Nombre total d'ouvrages } \\
(\% \text { sur l'ensemble du } \\
\text { corpus) }\end{array}$ & $\begin{array}{c}34 \\
(22,1 \%)\end{array}$ & $\begin{array}{c}28 \\
(18,2 \%)\end{array}$ & $\begin{array}{c}43 \\
(27,9 \%)\end{array}$ & $\begin{array}{c}26 \\
(16,9 \%)\end{array}$ & $\begin{array}{c}23 \\
(14,9 \%)\end{array}$ & $\begin{array}{c}154 \\
(100 \%)\end{array}$ \\
\hline $\begin{array}{l}\text { Scolarisation (\% dans le } \\
\text { type de handicap) }\end{array}$ & 15 & 20 & 29 & 15 & 11 & 90 \\
\hline $\begin{array}{l}\text { Scolarisation + MS (\% } \\
\text { dans le type de handicap) }\end{array}$ & $(44,1 \%)$ & $(71,4 \%)$ & $(67,4 \%)$ & $(57,7 \%)$ & $(47,8 \%)$ & $(58,4 \%)$ \\
\hline $\begin{array}{l}\text { Scolarisation + MS } \\
+ \text { famille (\% dans le type } \\
\text { de handicap }\end{array}$ & 2 & 4 & 1 & 4 & 5 & 17 \\
$(14,3 \%)$ & $(2,3 \%)$ & 1 & $(15,3 \%)$ & $(21,7 \%)$ & $(11 \%)$ \\
\hline
\end{tabular}

Si chaque type de handicap est plutôt bien représenté (de 23 à 43 ouvrages, soit respectivement de 14,9 à $27,9 \%$ de l'ensemble), la question de la scolarisation de façon générale est abordée par, en moyenne, moins des deux tiers des ouvrages $(58,4 \%)$ et la scolarisation en MS est traitée elle-même de façon plutôt marginale et hétérogène $(11 \%$ en moyenne et de $2,3 \%$ pour le handicap locomoteur à $21,7 \%$ pour l'autisme).

\section{L'analyse des ouvrages par type de handicap}

Dans les trois ouvrages sur la déficience visuelle, il y a peu d'éléments sur le MS, mais les enfants semblent s'y sentir bien. Malgré son récent déménagement, Stella [1], 13 ans, souhaite rester dans son " école spécialisée ", même si les trajets impliquent une aide que son nouveau jeune voisin Miro va proposer. Hugo [2], jeune orphelin, se plaît et a des amis en institut pour personnes avec cécité, mais il souhaite quitter l'institut pour être avec des personnes voyantes, et l'ouvrage relate ses efforts de travail et son combat pour se faire accepter. Mary [3], 9 ans, " presqu'aveugle » cherche à conquérir son autonomie face à des parents et une grand-mère surprotecteurs. Seul un évènement exceptionnel - son enlèvement - à l'issue heureuse lui ayant permis de montrer audace et courage transformera le regard de ses parents.

La présence de la famille est peu marquée dans le premier récit, elle est inexistante dans le second, apparaît davantage dans le troisième où les parents et la grand-mère ne considéreront plus Mary " comme un bébé » après son enlèvement.

Les données sur les relations famille-école restent ténues: le père de Stella par exemple essaie de trouver une aide pour l'aménagement des trajets plutôt que recourir à l'internat mais sans approfondissement de la réflexion sur le choix opéré.

Les leviers de l'inclusion avec l'école restent eux aussi effleurés. Ignorés dans le troisième ouvrage qui se limite à une brève allusion soulignant les représentations discriminantes des enfants voyants: "Tu vas dans une école spécialisée, non ? Là où vont les débiles et les monstres ? ", ils renvoient au seul recours à un ami dans le premier (Miro, tombé amoureux de Stella, se propose pour l'accompagner dans ses trajets). Hugo enfin, victime des moqueries des autres élèves, est lui aussi soutenu par son amie Aïssata qui partage l'expérience du rejet. C'est le seul des trois ouvrages où un enseignant s'implique directement dans une démarche médiatrice.

La scolarisation en MS n'apparaît pas réellement centrale dans les quatre ouvrages consacrés à la surdité : la thématique conductrice est plutôt celle de la LSF (Langue des Signes Française). Résultant d'un choix d'abord parental, elle mise en œuvre au plan éducatif et pédagogique de façon individualisée et adaptée au degré de surdité de chaque élève et de ses compétences, 
avec des enseignants bienveillants qui font progresser les enfants et leur permettent de reprendre confiance en eux ; elle favorise, voire structure les relations familiales et sociales.

Les parcours de chaque enfant, de chaque famille ont pu être différents : Horacio [4] est l'objet d'une surdité acquise ; ayant été scolarisé en milieu ordinaire, il refuse d'abord d'intégrer l'institut mais finalement s'y épanouit. Les parents de Lisa [5] ont tous deux une surdité et la mère garde un souvenir traumatisant d'une oralisation forcée. Chez Mattews [6], scolarisé en MS dès 2 ans, toute la famille (père, mère et sœur) apprend la LSF et la mère est très vigilante à ce que son fils puisse toujours suivre les conversations. La LSF apparaît bénéfique, créant un climat favorable tant en classe ( " dans un monde à nous, gai ») qu'en famille, permettant de communiquer [4] [5] [7], de se faire des amis [5] [6], voire présente des fonctions réparatrices [7]. Concernant les relations intrafamiliales, l'ambivalence de la sœur de Jessica [6] est abordée, à la fois sa colère, sa honte, sa honte d'avoir honte, mais aussi sa fierté.

Dans le cadre des relations école-famille, les parents des quatre ouvrages valorisent l'institut et les apprentissages qui y sont réalisés, voire même par une collaboration directe [6] lorsque la mère initie la réalisation d'un spectacle. Dans trois livres, de jeunes camarades se révèlent vecteurs d'inclusion, toujours avec la médiation de la LSF telle cette adolescente mutique du fait d'un traumatisme [7] qui communiquera avec le jeune héros avec surdité et deviendra son amie puis son amoureuse.

Dans le seul ouvrage sur la déficience locomotrice, Théo [8], 12 ans, en situation de handicap depuis sa naissance se déplace en fauteuil roulant. Il est dépendant pour les moindres gestes de la vie quotidienne et vit en institution. II traverse une période de crise (chute de résultats scolaires, attitudes d'opposition, refus des soins spécialisés...) qui ne relève pas tant de la qualité d'accueil de l'institution que de la confrontation de Théo à son sentiment de dépendance et de gratitude obligée, et du non-dit familial sur l'histoire de son handicap.

Si Théo se sent coupable de ses revendications à sortir de l'institution en raison des problèmes que cela poserait en particulier pour sa mère, le lien entre la famille et l'institution s'organise en véritable partenariat; en particulier trois professionnels de l'institution (le professeur de sport, la professeure principale et la psychologue) vont favoriser l'autonomie de Théo en travaillant avec les parents (permettant des activités hors institution). La psychologue avec qui l'enfant arrive à mettre en mots son mal-être, va être une grande ressource, car elle favorisera la prise de conscience par les parents de l'importance d'aborder avec leur fils les non-dits liés à sa naissance et la façon dont ils ont vécu son handicap.

Les enfants avec trisomie 21 sont présentés, dans deux ouvrages sur trois, comme des enfants qui se plaisent dans la structure au sein de laquelle ils font des activités variées. Laurette [9] est scolarisée en IMP (Institut Médico-Pédagogique) appelé le « centre Sourire ». " Le centre, c'est une école spécialisée pour les enfants comme moi » dit-elle. Elle y fait toutes sortes d'activités comme de la peinture, de la musique ou du poney. Les difficultés internes d'inclusion caractérisent les deux autres ouvrages : Florent [11] est accueilli en institution, probablement en IMP. II peut s'emporter et avoir des colères. Les difficultés d'inclusion, le regard des autres et les moqueries ainsi que leurs conséquences, mais aussi le mal-être que cela engendre pour son frère Clément, sont au cœur de l'ouvrage. II y a peu de lien ou de passerelles entre MS et MO dans ces deux ouvrages. Très peu d'éléments sont par exemple mentionnés concernant la scolarisation de Florent à l'institut, si ce n'est le pointage d'une intégration compliquée : Florent refuse de se rendre à l'IMP et les professionnels sont mis à mal par ses difficultés de comportement. Les difficultés intellectuelles d'Éric [6] scolarisé en IMPRO (Institut MédicoProfessionnel) sont crûment décrites, mais ses capacités sont mises en avant. Les défauts de qualité d'accueil sont pointés, le directeur mentionne une évolution " désespérante " sur le ton de la dérision. Le père se dit exaspéré par Éric, ses manies, ses rituels, il a du mal à " accepter » le handicap de son fils, qu'il décrit comme un " coucou » qui prend toute la place dans le nid, 
« un accroc dans leur vie » et les activités réalisées à l'institut sont dénigrées. L'IMPRO n'est donc pas très valorisé dans les apprentissages et les progrès scolaires qu'il tente de mettre en place, mais suite à une fugue d'Éric lors d'un séjour organisé par l'IMPRO, le père nouera une complicité avec son fils s'appuyant sur les compétences issues des apprentissages professionnels réalisés dans l'institution.

De son côté, le "très gentil » directeur de l'IMP propose que Laurette passe une journée d'inclusion par semaine en école ordinaire pour favoriser ses progrès. La maman appréhende cette inclusion, mais elle donne son accord pour tenter l'expérience dans l'école qui accueille également la sœur cadette. La maîtresse soutient l'inclusion, estime que "cela se passe très bien », mais les parents des autres élèves s'y montrent opposés, craignant que le programme scolaire de leur enfant soit ralenti. Grâce aux apprentissages réalisés à l'IMP, notamment ses compétences en équitation, Laurette est valorisée et elle se fait de nombreux amis. L'ouvrage dont Nicolas est le héros [10] traite le MS via un hôpital de jour, solution rarement proposée pour des enfants avec trisomie. II met en exergue de façon très pertinente les difficultés d'inclusion en $\mathrm{MO}$, les difficultés pour trouver des solutions d'accueil en MS, les tensions que cela peut engendrer avec les institutions manquant de places adaptées et leurs répercussions sur la famille (stress et inquiétude des parents et de la fratrie pour l'avenir).

Concernant les liens famille-institution, si les parents sont présents, la thématique spécifique des liens fraternels est mise en avant : frères et sœurs sont responsabilisés, voire parentalisés, et font le plus souvent lien avec l'inclusion en MO.

Elsa, sœur cadette de Laurette, est dans une position difficile puisque l'une et l'autre sont dans la même école en niveaux inversés par rapport à leur âge réel. Pourtant, Elsa défend vivement l'inclusion de Laurette envers ses pairs. À l'hôpital de jour, Nicolas a des qualités d'empathie importantes envers ses pairs qui attendrissent l'infirmière, mais son frère Étienne envisage l'avenir de Nicolas avec un pronostic réservé et il partage explications et préoccupations avec une amie. La famille, très présente, prépare Étienne à ses futures responsabilités vis-à-vis de son frère. La responsabilisation de Clément, frère de Florent, et sa difficulté à faire face aux réactions des autres sont aussi mises en exergue.

Les ouvrages sur l'autisme ne proposent pas d'information sur la nature du MS, même lorsqu'il est mentionné. Marc [13] a fait l'objet d'une tentative de démarche d'inclusion avec le soutien bienveillant de sa maîtresse, mais la démarche est mise en échec du fait de l'opposition exprimée par les parents des autres enfants de l'école ; dès lors, le MS résulte d'une obligation par défaut. Achille [14] lui aussi voit sa scolarisation en MO compromise dans sa continuité malgré son envie, et les centres d'accueil comme solution de recours ne sont que mentionnés. Aliénor [15] rend la vie de famille difficile du fait de l'intensité de ses troubles ; le projet d'accueil en MS est présenté comme une solution de "sortie de tunnel ». La scolarisation d'Adrien [4] en MS, solution qui semble être d' "évidence » - n'est jamais présentée dans sa réalité. Susan [17] est accueillie dans une institution semble-t-il de type IMP, dont les professionnels eux-mêmes sont mis en difficulté par l'enfant et seul un projet d'accueil en institut spécialisé adapté trouve grâce aux yeux des parents découragés.

Les familles apparaissent donc ici en difficulté psychologique et matérielle. Les parents sont en souffrance, voire " à bout de souffle "; ils ne croient plus eux-mêmes à l'inclusion en MO. L'accueil en MS relève dès lors d'un non-choix par rapport au MO inaccessible du fait du peu de tolérance des autres parents ou de manque de moyens éducatifs. La fratrie est mise à mal, en souffrance également, pouvant faire preuve d'un grand dévouement, voire d'une abnégation, dont le soulagement ne s'opérera que par l'accueil en institution de l'enfant avec autisme.

Les enseignants apparaissent présents et constructivement actifs lors de démarches inclusives, même lorsqu'elles sont mises en échec, mais lorsqu'il s'agit d'institutions spécialisées, seules leurs difficultés de " prise en charge » (manque de places, éloignement) sont pointées. 


\section{Synthèse et discussion}

Comment se reflète l'évolution de la société et des dispositifs de scolarisation dans les ouvrages analysés?

D'abord indiscutablement il y a eu en ce début de XXlè siècle une évolution quantitative et qualitative de la littérature de jeunesse (Schneider, Mietkiewicz, 2013). La question du handicap est devenue dans ce contexte une thématique enrichie et cette évolution a favorisé même l'idée d'une " littérature inclusive » (Beckett, Elliso, Barreth, Shah, 2010 ; Matthew, Claw, 2007).

Nous avons ici fait d'abord le constat suivant: par comparaison avec le MO (Lemoine, Mietkiewicz, Schneider, 2017), la scolarisation en MS est peu présente en nombre dans les ouvrages. Le corpus, de petite taille, est réparti de façon non homogène (un livre pour le handicap locomoteur jusqu'à cinq pour l'autisme), il ne représente que $11 \%$ de l'ensemble des ouvrages qui abordent la question du handicap, alors que plus de $58 \%$ des ouvrages abordent la scolarisation en MS.

Lorsque le thème est abordé ou mentionné, les parents ont plutôt des relations distantes avec le MS, peu inscrites dans un projet.

Le MS est présenté comme plutôt " neutre » dans les livres sur la cécité : on relève peu d'éléments sur le MS lui-même, sur le ressenti des enfants ou leurs difficultés, sur la famille sinon qu'elle est surprotectrice, et pas grand-chose enfin sur les liens parents-école (sans connotations négatives cependant). Les scénarios mettent plutôt l'accent sur les capacités et la volonté de l'enfant avec handicap, sa force de caractère, et la construction d'un lien électif avec un pair de même âge combinant des qualités complémentaires, ce qui permet une symétrie relationnelle.

Le MS est abordé de façon très positive pour la surdité, mais il s'agit au final d'un MS adapté et individualisé promouvant d'abord la LSF : les enfants s'y sentent bien, car ils communiquent avec les autres grâce à la LSF. Les familles se mobilisent et apprennent la LSF (parents et fratrie) pour communiquer avec leur enfant, et incluent l'entourage amical et social dans cette démarche, les méthodes d'apprentissage de l'institution - notamment de la LSF - sont validées par les parents.

Notons que trois ouvrages portant sur la déficience sensorielle $[2,6,7]$ font le parallèle avec le racisme pour parler de l'exclusion des enfants avec cécité [2] et surdité [6,7], le sentiment d'injustice et la profonde tristesse qu'elle entraîne chez les enfants, comme si l'empathie passait par l'expérience du rejet partagé.

L'ouvrage consacré au handicap locomoteur met en évidence que le MS propose des activités adaptées - par exemple le sport - qui favorisent l'autonomie et la confiance en soi, dont la carence chez ce jeune adolescent sous-tendait les difficultés d'adaptation initiales au MS. L'institution et ses professionnels contribuent aussi à son autonomie et à son bien-être psychologique par leur médiation avec les parents en permettant la levée des non-dits sur l'histoire familiale autour du handicap.

Même s'ils sont parmi les plus nombreux dans le corpus, on peut estimer que peu d'ouvrages sur la trisomie et l'autisme abordent la question de la scolarisation de l'enfant avec handicap en lien avec une institution spécialisée, alors que les enfants porteurs de ces troubles sont encore aujourd'hui les plus concernés par le MS (par rapport au handicap moteur ou sensoriel) où certains d'entre eux passent une grande partie de leur vie scolaire et professionnelle en institution. Une partie de l'explication résulte sans doute de la stratégie scénaristique des ouvrages, où le héros-narrateur est souvent le frère ou la sœur du jeune avec handicap, et l'histoire se situe alors plus volontiers en famille ou en relation avec les pairs au sein de l'école en MO. Mais si la question de la fratrie est d'importance (Lemoine, Mietkiewicz, Schneider, 2016), cette approche souligne d'autant le peu d'informations proposées aux jeunes lecteurs sur le MS de l'enfant porteur de handicap. Lorsqu'il est abordé, ce MS est présenté cependant de façon positive pour les livres sur la trisomie ; il est décrit comme proposant des activités variées même 
si peu de passerelles sont mentionnées entre MS-MO, les enfants s'y plaisent, les relations familiales se structurent autour d'une fratrie parentalisée, qui fera le levier vers l'inclusion en MO. Pour les familles d'enfant avec autisme, il apparaît d'abord comme un non-choix face au MO inaccessible, il est peu décrit sinon négativement, les parents (ou la fratrie) sont en grande souffrance, et les parents sont défaitistes par rapport à l'inclusion.

\section{Qu'est-ce que cette littérature peut apporter au lecteur?}

On constate donc que, si les ouvrages approchent de façon informée le handicap dans ses manifestations les plus tangibles, ils ne prennent en compte que partiellement le point de vue de l'enfant avec handicap dans la concrétude de sa relation au MS, donc la variété de ses milieux de vie. Les familles sont parfois peu présentes ou démunies face à l'adversité, comme s'il s'agissait de mettre en valeur les ressources de l'enfant héros, qu'il s'agisse de l'enfant avec handicap ou de la fratrie.

Mais le lecteur n'est pas un lecteur abstrait, c'est un lecteur incarné dans un contexte. On peut dès lors avoir le sentiment que les ouvrages traitant de l'accueil en MO visent au final davantage les pairs que l'enfant lui-même puisqu'ils manquent pour partie à saisir la diversité de ses expériences. En abordant la question des aides et des médiations offertes aux familles à travers des supports diversifiés, Joselin et Scelles (2014) mettent l'accent sur la question du « passage entre institutions " (ordinaires et spécialisées), des conditions de son accompagnement, des difficultés éprouvées par l'enfant en situation de handicap « qui n'aura pas entendu par ses aîné(e)s, ses cousin(e)s, ses camarades d'école, lui raconter comment se passe le passage en institution spécialisée » (p. 152). À certains égards, ces ouvrages n'atteignent que partiellement leur objectif puisqu'ouvrir une sensibilité au handicap doit s'appuyer certes sur le reflet des ressentis du jeune lecteur valide, l'autorisant à partager ses affects positifs et négatifs, mais aussi étayer le mouvement d'empathie par une sensibilisation aux éprouvés et expériences du sujet porteur de handicap. La question des pairs se révèle par exemple ici d'autant d'importance que l'on a montré la forte corrélation entre liens fraternels (inaugurant la "fratrie éternelle », selon l'expression de Poujol et Scelles, 2014) et les liens sociaux - lorsque les premiers sont entravés, le réseau social se restreint - donc la nécessité de se préoccuper de ces liens avec les pairs dès la petite enfance.

Cette lecture, sans doute critique, mérite cependant un point d'attention, une nuance et une ouverture qui doivent être mentionnés. Par notre approche, nous avons souligné la spécificité des scénarios et des représentations par type de handicap. Nous renvoyons le lecteur aux travaux de Josselin $(2008,2009,2013)$ qui pointent bien les effets de catégorisation que mobilisent chacun d'entre eux. Mais plusieurs thématiques importantes sont abordées dans plusieurs ouvrages : les tentatives d'inclusion et leurs aléas, tels les réactions des autres familles, l'ambivalence des enfants avec handicap quant à leurs choix d'orientation, leurs ressources personnelles, le travail psychique parental pour faire face au handicap, les relations intrafamiliales, les relations complexes avec le milieu éducatif, parfois peu protecteur mais aussi impliqué et soutenant. Audelà des effets réducteurs de la thématisation/catégorisation par handicap, le lecteur, enfant ou éducateur concerné par un handicap, est invité à aller chercher hors de la sphère de ce handicap spécifique les ressources scénaristiques qu'offre la littérature jeunesse. La distanciation identificatoire avec sa propre situation peut d'ailleurs contribuer à renforcer le filtre et autoriser le recul indispensable pour se positionner face aux situations évoquées (Léon, 2004) et ouvrir au dialogue.

\section{Conclusion}

L'inclusion ne repose pas sur une opposition entre MO et MS, mais sur leur articulation en fonction des projets des enfants, de leurs besoins, et de leur trajectoire développementale dans 
le cadre parfois d'une scolarisation mixte. Quelle que soit l'institution (MS-M0), le lien parentinstitution apparaît capital pour que l'enfant s'épanouisse et puisse s'inscrire dans son projet où s'articulent les deux microsystèmes institution et famille et y être acteur. Notre analyse des ouvrages de jeunesse consacrés au MS montre à la fois certaines limites en fonction des contenus véhiculés et des modèles proposés aux enfants relatifs à eux-mêmes et à leur entourage familial et institutionnel, mais elle souligne aussi les potentialités éducatives que contribue à mettre à jour le décryptage des ouvrages. Dans la clinique, enfants, adolescents et adultes en situation de handicap sont de plus en plus écoutés (cf. " patients experts ", " autoreprésentants ॥...). La littérature de jeunesse offre un potentiel d'appui indiscutable qui ouvre à l'exploration du point de vue subjectif de la personne en situation de handicap, il reste à développer des recherches sur l'usage effectif de cette littérature dans le cadre de politiques inclusives, cette démarche n'en est qu'à ses linéaments.

\section{Annexe : Corpus}

\section{Cécité :}

[1] FERDJOUKH M. 1996. La fille d'en face, Paris, Bayard, 2002.

[2] LENAIN T. 1997. Loin des yeux, près du cœur, Paris, Nathan, 2005, 2009.

[3] WILSON J. 2002. Le couteau sous les yeux, Paris, Hachette Jeunesse.

\section{Surdité :}

[4] DIAZ G. C. 2008. Ecoute-moi avec les yeux, Nantes, Gulf Stream Éditeur.

[5] JEAN D. 1999. Deux mains pour le dire, Albussac, 2 Vives Voix Éditions, 2004, 2006, 2015.

[6] MARTIN A. M. 1990. Le club des Baby-Sitters. Nos plus grands défis, Le langage secret de Jessica, Paris, Gallimard Jeunesse, 2000, 2006.

[7] YZAC A. 2006. L'enfant à la bouche du silence, Bruxelles, Alice Jeunesse.

\section{Handicap moteur :}

[8] LE GOUIC-PRIETO C. 2008. Non merci, Paris, Bayard Jeunesse.

\section{Trisomie 21:}

[9] CADIER F, GIREL S. 2006. Qui est Laurette ?, Paris, Nathan Poche, coll. " C'est la vie ».

[10] DEMEYERE-FOGELGESANG C. 2004. Étienne et Nicolas. Mayenne, Hachette Jeunesse, coll. " Histoires de vies ».

[11] PAQUELIER B. 2009. Tous les matins, je regarde passer les filles, Paris, Oskar Jeunesse, coll. « Poche Roman ».

[12] VENDAMME P. 1993. Éric et Lucile. Paris, Syros jeunesse, coll. « Les uns les autres », 2001.

\section{Autisme :}

[13] BEAUDOIN J. 2009. Mon copain Marc, Paris, Oxalide, Kid's Corner.

[14] FOZ V. 2014. Les ailes du papillon : Un battement d'ailes peut-il vraiment changer le destin?, Paris, Oskar.

[15] JAOUI S. 2003. Je veux changer de sœur, Paris, Casterman Roman, Junior, 2010.

[16] LARCHEZ M. 2001. J'ai un copain autiste, Colmar, Les Éditions d'Alsace.

[17] MARTIN A.M. 2006. Le club des Baby-Sitters : Nos plus grands défis. Le défi de Kristy, Paris, Gallimard Jeunesse. 


\section{Bibliographie}

BECKETT A., ELLISON L., BARRETT S., SHAH S. 2010. «'Away with the fairies?' Disability within primary-age children's literature», Disability \& Society, 25, 3, 373-386,

DEEVANSA-AGUILAR C. 2019. Rapport de la rapporteuse spéciale sur les droits des personnes handicapées, Nations Unies, Conseil des droits de l'homme, quarantième session.

FAIVRE H. 2006. " Du parent "pathogène" au parent partenaire », dans La nouvelle revue de l'adaptation et de la scolarisation, 2 (34), 23-34.

JOSELIN L. 2008. Les représentations du handicap dans la littérature jeunesse française et italienne, Unpublished doctoral dissertation, Université de Rouen, France.

JOSELIN L. 2013. " Représentations plurielles des héros déficients dans la littérature jeunesse », dans B. Schneider \& M.-C. Mietkiewicz (dir.). L'enfant dans les livres. Représentations, savoirs, normes (pp. 103-115), Toulouse, Erès.

JOSELIN L, SCELLES R. 2014. "Rites de passage et handicap, un apprentissage parental partagé », dans La revue internationale de l'éducation familiale, $\mathrm{n}^{\circ} 36,141-160$.

LEMOINE L, MIETKIEWICZ M.-C, SCHNEIDER B. 2016. "Fratrie et handicap (trisomie 21 versus autisme): les représentations dans la littérature jeunesse », dans Revue Francophone de la Déficience intellectuelle, vol. 27, 141-162.

LÉON R. 2004. La littérature de jeunesse à l'école : Pourquoi ? Comment?, Paris, Hachette.

MATTHEW N, CLOW S. 2007. «Putting disabled children in th pisture ; protting inclusive children's book and media ", International Journal of Early Childhood, 39, 2, 65-78.

MIET P, Recher S. 1989. « Des livres, des images, des absents, dans L'enfant handicapé à travers la littérature enfantine », Paris, CNDP, p. 7-15.

MIETKIEWICZ M.-C, LEMOINE L, SCHNEIDER B. 2017. « Enfant en situation de handicap et scolarisation en milieu ordinaire: quelle place pour la famille? Une approche par la littérature jeunesse ", dans Psychologie et éducation, $n^{\circ} 4$, 29-52.

POUJOL A.-L, SCELLES R. 2014. "Le point de vue des adultes avec une trisomie 21 sur leurs relations fraternelles et aux pairs extrafamiliaux: impact sur leur vie intrapsychique et intersubjective», Pratiques psychologiques, 20, 83-94.

SCHNEIDER B. 2017. "Numéro thématique: A l'école de la littérature jeunesse », dans Psychologie et éducation, 4.

SCHNEIDER B, MIETKIEWICZ M.-C. 2013. Les enfants dans les livres. Représentations, savoirs, normes, Toulouse, Érès. 